apuntesuniversitarios.upeu.edu.pe

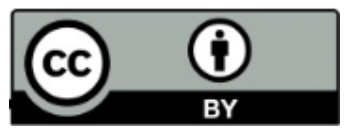

Apuntes Universitarios, 2021: 11(2), abril-junio ISSN:

2304-0335 DOI: https://doi.org/10.17162/au.v11i2.632

\title{
Diseño y validez de contenido de una rúbrica analítica socioformativa para evaluar competencias investigativas en posgrado
}

\author{
Design and content validity of a socioformative rubric to evaluate \\ investigative skills in postgraduate
}

\author{
Alicia Agromelis Aliaga-Pacora ${ }^{1 a}$, Luis Gibran Juárez-Hernández², Raúl Herrera-Meza ${ }^{3}$ \\ Universidad Nacional Federico Villarreal, Perú- EUPG ${ }^{1}$ \\ Centro universitario CIFE, México ${ }^{23}$
}

iD Orcid ID: https://orcid.org/0000-0002-4608-2975

(iD) Orcid ID: https://orcid.org/0000-0003-0658-6818

(iD) Orcid ID: https://orcid.org/0000-0003-2605-4878

Recibido: 21 de octubre 2020

Aceptado: 07 de diciembre de 2020

\begin{abstract}
Resumen
En la presente investigación, se diseñó y validó en contenido un instrumento para evaluar competencias investigativas en posgrado. Para su diseño se integraron aspectos evidenciados en el desarrollo del trabajo de tesis. El instrumento fue revisado por cuatro expertos para el análisis de validez de facie y para el análisis de validez de contenido se efectuó un juicio de expertos. Concluidas estas fases se realizó un pilotaje del instrumento con 38 asesores para evaluar el grado de comprensión de instrucciones e ítems, así como la confiabilidad del instrumento. El instrumento se diseñó como rúbrica analítica socioformativa integrando cinco aspectos del desarrollo del trabajo de tesis a través de 11 ítems y para cada uno de ellos cinco niveles de desempeño con un descriptor propio. Los expertos expresaron sugerencias de redacción para optimizar la rúbrica. Los jueces expertos validaron en contenido los ítems (V de Aiken>0.80; VI del IC al 95\%>0.75). El pilotaje mostró un buen grado de comprensión de las instrucciones e ítems y relevancia de las preguntas y se determinó que el instrumento posee confiabilidad (Alfa de Cronbach: 0.832). Se presenta un instrumento válido en contenido, sin embargo, se requiere proseguir con el análisis de sus propiedades psicométricas (validez de constructo y confiabilidad).
\end{abstract}

Palabras clave: Rúbrica socioformativa; competencias investigativas; validez; confiabilidad; juicio de expertos.

${ }^{\mathrm{a} C}$ Correspondencia al autor:

E-mail: aliciaaliagapacora@gmail.com 


\begin{abstract}
In the present investigation, an instrument for evaluating postgraduate research competencies was designed and validated in content. For its design, aspects evidenced in the development of the thesis work were integrated. The instrument was reviewed by four experts for the facie validity analysis and an expert judgment was made for the content validity analysis. At the end of these phases, an instrument was piloted with 38 advisers to assess the degree of understanding of instructions and items, as well as the reliability of the instrument. The instrument was designed as a socio-formative analytical rubric integrating five aspects of the development of the thesis work through 11 items and for each of them five levels of performance with their own descriptor. The experts expressed writing suggestions to optimize the rubric. The expert judges validated the items in content (Aiken's V> 0.80; LV CI 95\%>0.75). The piloting showed a good degree of understanding of the instructions and items and relevance of the questions and it was determined that the instrument has reliability (Cronbach's Alpha: 0.832). A valid content instrument is presented; however, it is necessary to continue with the analysis of its psychometric properties (construct validity and reliability)
\end{abstract}

Keywords: Socioformative rubric; investigative competencies; validity; reliability; expert judgment.

\title{
Introducción
}

En el ámbito de estudios de posgrado, desarrollar las competencias investigativas es clave en la formación como investigador, ya que permite identificar problemas del contexto, vincular habilidades, destrezas y saberes para emplearlos en la vida cotidiana (Luque et al., 2018); además, es la piedra angular que sustenta el binomio universidad-sociedad (Reiban et al., 2017; Sergio Tobón, 2008).

En este contexto, los estudiantes de posgrado deben desarrollar sus habilidades y destrezas de investigación para "que se conviertan en verdaderos promotores de cambios significativos en los procesos productivos de su país" (Moros-Briceño, 2018, p.52), y se apropien de las competencias de investigación, con la resolución eficaz de problemas, ya que la investigación científica es inherente a la calidad de las universidades. Ella representa la cultura investigativa de dicho claustro (Victoria et al., 2018). En la misma línea de pensamiento, diversos autores (Balbo, 2000; Gheller, 2002; Guajardo et al., 2011; Mgquba \& Underwood, 2016; Rizo-García, 2012; Tapia et al., 2018; Verhoeven et al., 2016), precisan que en posgrado las competencias investigativas son determinantes en la construcción, creación y transmisión de conocimientos que les permite contextualizar el problema en cualquier realidad que se encuentren.

No obstante la importancia del desarrollo de las competencias investigativas, aun son pocos 
los referentes y propuestas metodológicos indicados para su desarrollo y su evaluación (CamposCéspedes et al., 2012; Cardoso \& Cerecedo, 2019; Estrada, 2019; George-Reyes \& RamírezMartinell, 2019; Grijalva-Verdugo \& Zazueta, 2017; Gutiérrez-Castillo et al., 2017; Tapia et al., 2018; Valdés-Cuervo et al., 2012). Los instrumentos referidos enfatizan la evaluación de la capacidad de manejo de las TIC, búsqueda de información y metodología, destacando que únicamente las propuestas de Valdés-Cuervo et al. (2012) y Tapia et al. (2018) consideran seriamente el proceso de los trabajos de investigación. Otro aspecto en común es el tipo de instrumento empleado, el cual en su mayoría son de tipo cuestionario con respuestas en escala tipo Likert.

Ante esta situación, evaluar competencias investigativas desde el enfoque socioformativo implica el uso de rúbricas. En este sentido, es importante señalar que las competencias son el conjunto de actuaciones integrales que identifican, interpretan, argumentan y dan soluciones a los problemas de la realidad, haciendo uso de los cuatro tipos de saber: conocer, ser, convivir y hacer. Relacionando estos con idoneidad, mejora continua y ética, implica que el discente no solo reconozca el problema, sino que plantee soluciones de forma holística, que involucre un trabajo multidisciplinario, cooperativo y el trabajo con pares y expertos "fortalece la disposición de integrar y formar equipos científicos como resultado de una formación científica profesional" (Ceballos-Almeraya \& Tobón, 2019, p. 14).

Desde este mismo enfoque se denota el uso de las rúbricas, que es definida como una herramienta de evaluación que faculta valorar el desempeño en la resolución de problemas reales del entorno, relacionando una serie de indicadores con descriptores de niveles de dominio (Hernández-Mosqueda et al., 2016). Por ello, la necesidad de sumar un instrumento que evalué los procesos formativos en el ámbito académico y establecer sistemáticamente la mejora continua del estudiante como es el caso de la rúbrica que facilita la autoevaluación a través rangos o niveles de desempeño (Díaz-Barriga, 2011); es decir, una rúbrica analítica socioformativa presenta descriptores de nivel de dominio (pre-formal, receptivo, resolutivo, autónomo y estratégico); existen algunos instrumentos para evaluar las competencias investigativas en estudiantes de pregrado (Cardona-Arias, 2019; Ceballos-Almeraya \& Tobón, 2019; Vázquez-Antonio et al., 2018).

De acuerdo con el estudio efectuado se detectó que era necesario construir un instrumento para evaluar competencias investigativas en el nivel de posgrado. Al respecto, la Universidad 
Nacional Federico Villareal, lamentablemente no cuenta con instrumentos idóneos para valorar gradualmente las competencias investigativas; siendo estos meros cuestionarios y listas de verificación compuestos por una serie de indicadores que no permiten a los asesores identificar la calidad y el progreso de los estudiantes, ni el desarrollo de sus competencias investigativas, debido a que dichos métodos no presentan descriptores para los diferentes niveles posibles (Marin-Garcia et al., 2015; Tobon et al., 2020). Por ende, los niveles educativos de posgrado deben estar encaminados a la promoción del desarrollo de competencias investigativas que permitan generar nuevos conocimientos desde una visión científica, a fin de alcanzar la verdad, dentro de la sociedad del conocimiento y haciendo uso de la evaluación formativa (Fraile et al., 2017; MartínezRodríguez \& Márquez-Delgado, 2014).

De acuerdo con lo anterior, en el presente estudio se establecieron las siguientes metas: 1) diseñar un instrumento en forma de rúbrica analítica socioformativa para evaluar competencias investigativas desde el enfoque socioformativo y 2) efectuar un juicio de expertos al instrumento para el análisis y determinación de su validez de contenido.

\section{Metodología}

El presente trabajo comprende un estudio de tipo instrumental, debido a que consiste en la construcción de una herramienta de evaluación, y análisis de sus propiedades psicométricas (Montero et al., 2017), en específico en el presente se analizará la validez de contenido y confiabilidad.

\section{Procedimiento}

El estudio instrumental consideró las siguientes etapas:

1). Diseño del instrumento "Rúbrica socioformativa para evaluar competencias investigativas en posgrado". De manera inicial se realizó una búsqueda de referentes teóricos sobre las competencias investigativas de forma que se identificaron aspectos considerados como las competencias genéricas en investigación, desarrollo de las competencias en el proceso de investigación, diseño de investigación, instrumentales, personales, gestión para la divulgación, dominio de metodología de la ciencia, conocimiento teórico del campo profesional, nociones de diseño, aplicación y evaluación de instrumentos de recolección de datos, uso de bases científicas de datos, saberes estadísticos, interpretación y criterios cualitativos, redacción de informes y reportes de investigación, competencias cognitivas, formulación de preguntas, competencias 
observacionales, competencias, procedimentales.

Posteriormente se realizó una búsqueda de antecedentes instrumentales, encontrándose una serie de instrumentos para la evaluación de competencias investigativas en posgrado (Cardoso \& Cerecedo, 2019; George-Reyes \& Ramírez-Martinell, 2019; Grijalva-Verdugo \& Zazueta, 2017; Tapia et al., 2018), denotando que estos referentes instrumentales en su mayoría emplean el cuestionario como instrumento. En esta búsqueda también se encontraron algunos aportes que utilizaron las rúbricas socioformativas como instrumento para medir competencias investigativas, pero en el nivel de pregrado (Cardona-Arias, 2019; Ceballos-Almeraya \& Tobón, 2019; VázquezAntonio et al., 2018). En definitiva, no se encontró rúbricas analíticas socioformativas para evaluar las competencias investigativas en posgrado, las cuales se estiman de gran valor en el desarrollo de las competencias investigativas debido a que, ésta permite identificar los niveles específicos de logro (Tobón, 2017) . Ambas búsquedas (referentes teóricos e instrumentos) se realizaron en bases de datos relevantes (Google Scholar, Redalyc, Scielo, WoS, Scopus).

Luego de haber ejecutado el análisis, se diseñó el instrumento "Rúbrica analítica socioformativa para evaluar competencias investigativas en posgrado" evidenciados en el desarrollo del trabajo de tesis se conformó mediante los siguientes aspectos: (a) introducción, es la presentación del problema, antecedentes del tema, marco teórico, formulación del problema, objetivo e hipótesis y justificación de la investigación; (b) metodología, es la presentación del proceso a través del cual se hizo el estudio, indicando aspectos como tipo de investigación, participantes, procedimiento, instrumentos y técnica de análisis de los datos; (c) resultados, es la descripción de los hallazgos más importantes acorde con los propósitos del estudio, en forma de texto o mediante tablas o figuras; (d) informe final, consiste en la aplicación del estilo y el formato APA en su última edición y la redacción con precisión académico científica; y (e) trabajo colaborativo, consiste en la conexión con otros estudiantes y profesionales creando comunidades de aprendizaje; y 11 ítems. Cada uno de los aspectos es evaluado en cinco niveles y cada uno con sus descriptores que determinan con precisión el nivel, conforme a la taxonomía socioformativa: preformal (desempeño limitado o demasiado básico), receptivo (desempeño básico con alto requerimiento de asistencia por parte del asesor), resolutivo (desempeño aceptable y suficiente), autónomo (desempeño bueno y eficiente), estratégico (desempeño sobresaliente, creativo e innovador) (Tobón, 2017). 


\section{Tabla 1}

Aspectos y preguntas del Instrumento

\begin{tabular}{|c|c|}
\hline Aspectos & Preguntas del instrumento \\
\hline Introducción. & $\begin{array}{l}\text { 1. ¿En qué nivel el estudiante es capaz de identificar, describir y explicar un problema de } \\
\text { investigación de su contexto para proponer alternativas de solución? } \\
\text { 2. ¿En qué nivel el estudiante es capaz de recabar, seleccionar y organizar información } \\
\text { válida y confiable para la fundamentación del problema identificado? } \\
\text { 3. ¿En qué nivel el estudiante es capaz de formular las preguntas, objetivos y/o hipótesis } \\
\text { para seguir con el proceso de investigación? } \\
\text { 4. ¿En qué nivel el estudiante es capaz de construir el marco teórico integrando diferentes } \\
\text { campos del saber en su investigación? } \\
\text { 5. ¿En qué nivel el estudiante es capaz de exponer y argumentar la relevancia teórica, } \\
\text { metodológica y práctica de la investigación? }\end{array}$ \\
\hline Metodología & $\begin{array}{l}\text { 6. ¿En qué nivel el estudiante es capaz de seleccionar el diseño de investigación? } \\
\text { 7. ¿En qué nivel el estudiante es capaz de registrar y sistematizar los datos obtenidos en }\end{array}$ \\
\hline Resultados & $\begin{array}{l}\text { el trabajo de campo? } \\
\text { 8. ¿En qué nivel el estudiante es capaz de analizar los datos para estructurar la respuesta } \\
\text { a la pregunta de investigación? } \\
\text { 9. ¿En qué nivel el estudiante es capaz de interpretar los datos obtenidos para construir, } \\
\text { exponer y fundamentar los resultados de la investigación? }\end{array}$ \\
\hline Informe final & $\begin{array}{l}\text { 10. ¿En qué nivel el estudiante es capaz de redactar el informe final (Tesis) del trabajo } \\
\text { con precisión académico-científica (redacción y ortografía) y técnica (normas APA } \\
\text { vigente)? }\end{array}$ \\
\hline Trabajo colaborativo & $\begin{array}{l}\text { 11. ¿En qué nivel el estudiante es capaz de obtener colaboración y apoyo de parte de } \\
\text { personajes solventes profesional y académicamente para el desarrollo de su } \\
\text { investigación? }\end{array}$ \\
\hline
\end{tabular}

Fuente: elaboración propia

\section{2). Revisión por expertos}

El instrumento fue revisado por cuatro expertos (Tabla 2). Esta fase es referida como validez de prima facie y consiste en determinar la pertinencia y relevancia de los ítems concernientes al constructo y su redacción (Haynes et al., 1995; Koller et al., 2017; Mendoza Mendoza \& Garza, 2009) citado en (Martínez-Valdés \& Juárez-Hernández, 2019). En esta fase, los expertos podían realizar comentarios y sugerencias, correcciones, respecto a la redacción, pertinencia y relevancia de los ítems y descriptores de los niveles de desempeño propuestos. La elección de los expertos se realizó tomando en cuenta; grado de Doctor(a), mínimo grado de maestra(o), años de experiencia en el área de investigación, experiencia en la revisión y validación de instrumentos de evaluación, es decir una selección sostenida en el biograma propuesto por CIFE (2018) además contempla las variables cuantitativas concernientes a tiempo de experiencia en el área, experiencia en asesoría de tesis, experiencia en diseño, revisión y validación de instrumentos de evaluación. 
Tabla 2

Caracterización sociodemográfica de los expertos

\begin{tabular}{ll}
\hline \multicolumn{1}{c}{ Indicador } & Dato \\
\hline Sexo: & $50,0 \%$ hombres \\
& $50,0 \%$ mujeres \\
Roles: & Docentes: $100,0 \%$ \\
Último nivel de estudio: & Maestría: $50,0 \%$ \\
& Doctorado: $50,0 \%$ \\
& 18,0 promedio \\
Áreas de experiencia profesional $(\%)):$ & 9,5 promedio \\
Número de años de experiencia en investigación: & 5,5 promedio \\
Número de artículos publicados en el área: & 5 promedio \\
Número de ponencias presentadas en el área: & 3 promedio \\
Número de libros publicados en el área: & $98 \%$ \\
Experiencia en la revisión, diseño y/o validación de un & \\
determinado instrumento de investigación & \\
\hline Funte: elaboracion propia
\end{tabular}

Fuente: elaboración propia

\section{3). Análisis de validez de contenido}

Una vez atendidas las mejoras y observaciones de la revisión por expertos, se llevó a cabo el juicio de expertos con una perspectiva cualitativa y cuantitativa. La selección de los jueces se efectuó bajo los siguientes criterios; grado académico mínimo de maestría, años de experiencia en el área, y experiencia en el diseño y validación de instrumentos de investigación (JuárezHernández \& Tobón, 2018; Montoya-López \& Juarez-Hernández, 2019). El formulario en línea fue enviada a 28 expertos vía e-mail, de los cuales participaron 16 en la evaluación de la "Rúbrica analítica para evaluar competencias investigativas desde la socioformación" (Tabla 3). En cuanto al instrumento empleado para que los jueces realizaran la evaluación fue la escala de jueces expertos por CIFE (2018), que se compone de una sección cualitativa y una sección cuantitativa. Desde la perspectiva cualitativa, los expertos podían hacer recomendaciones y sugerencias en extenso respecto a la pertinencia y redacción de los ítems y descriptores. Por su parte desde la perspectiva cuantitativa los expertos evaluaron mediante una escala Likert (constituida de uno a cuatro valor más bajo y alto respectivamente) los criterios de pertinencia y redacción de los ítems y descriptores. El análisis de la evaluación cuantitativa se efectuó por medio del coeficiente de validez de contenido V de Aiken e intervalo de confianza al 95\% (Penfield \& Giacobbi, 2004), considerándose como valor mínimo de 0.80 para la aceptación de un ítem como válido (Merino Soto \& Livia Segovia, 2009) y de 0.60 para el valor inferior del intervalo. Con la confluencia de 
las dos evaluaciones anteriores, el instrumento se adecuó y optimizó.

\section{Tabla 3}

Caracterización sociodemográfica de los jueces expertos

\begin{tabular}{ll}
\hline \multicolumn{1}{c}{ Indicador } & Dato \\
\hline Sexo: & $43,8 \%$ hombres \\
Roles: & $56,2 \%$ mujeres \\
& Docentes: $87,5 \%$ \\
Último nivel de estudio: & Directivo: $12,5 \%$ \\
& Maestría: $18,8 \%$ \\
Áreas de experiencia profesional: & Doctorado: $81,2 \%$ \\
& Docente de posgrado $75 \%$ \\
Número de años de experiencia profesional: & Docente investigador $25 \%$ \\
& {$[12-20]=31,25 \%$} \\
Número de artículos publicados en el área: & {$[21-27]=37,50 \%$} \\
& {$[28-35]=31,25 \%$} \\
Número de ponencias presentadas en el área: & {$[0-3]=68,75 \%$} \\
& {$[4-6]=18,75 \%$} \\
& {$[7-13]=12,75 \%$} \\
Número de libros publicados en el área: & {$[0-3]=68,75 \%$} \\
& {$[4-6]=18,75 \%$} \\
Experiencia en la revisión, diseño y/o validación & {$[7-13]=12,75 \%$} \\
de un determinado instrumento de investigación & {$[0-2]=75,00 \%$} \\
\hline
\end{tabular}

Fuente: elaboración propia

\section{4). Aplicación de la prueba piloto}

Posteriormente el instrumento se aplicó a un grupo piloto conformado por 38 asesores de tesis de la Escuela Universitaria de Posgrado, de la Universidad Nacional Federico Villarreal del Distrito de Lima Cercado. Adicionalmente de la aplicación del instrumento, se utilizó un cuestionario de satisfacción (CIFE, 2018) con la finalidad de evaluar el grado de compresión de instrucciones e ítems y relevancia de estos últimos, así como efectuar un análisis de confiabilidad mediante el Alfa de Cronbach.

\section{Resultados}

\section{Revisión por expertos}

Los expertos sugirieron realizar precisiones en el contenido de los descriptores del ítem dos del instrumento propuesto, en el ítem tres sugirieron cambiar el término hipótesis de 
investigación por pregunta de investigación. En general expresaron sugerencias de redacción, antes mencionada, de algunos descriptores para optimizar la rúbrica, los cuales se tomaron en cuenta. A continuación, se presenta la Rúbrica Analítica.

\section{$\underline{\text { Juicio de expertos }}$}

Los resultados de la evaluación cuantitativa con respecto a pertinencia y redacción se evidenció la validez de los ítems formulados (V de Aiken>0.80; VI del IC al 95\%>0.75) (Tabla 4). Las observaciones y recomendaciones de los jueces denotan que la rúbrica socioformativa, en la mayoría de los ítems, son pertinentes y coherentes en la redacción. No obstante, hubo algunas sugerencias de mejora respecto a la redacción. Se sugirió precisar la redacción del ítem 2, eliminar o cambiar el termino ficha, decía "organizar en fichas, información válida y confiable para la fundamentación del problema identificado" se eliminó la palabra ficha. En el ítem 4 se sugirió cambiar el término estudio teórico por marco teórico. En el ítem 7 cambiar APA 7 séptima edición por vigente o actual. Este conjunto de sugerencias y correcciones se integraron al instrumento (Tabla 5).

\section{Tabla 4}

Análisis cuantitativo del juicio de expertos mediante la $V$ de Aiken y sus intervalos de confianza al 95\%

\begin{tabular}{ccccc}
\hline \multirow{2}{*}{ Ítem } & Pertinencia & IC al 95\% & Redacción & IC al 95\% \\
\hline 1 & 0.896 & $0.778 \pm 0.954$ & 0.958 & $0.859 \pm 0.988$ \\
2 & 0.979 & $0.890 \pm 0.996$ & 0.979 & $0.890 \pm 0.996$ \\
3 & 0.958 & $0.859 \pm 0.988$ & 0.896 & $0.778 \pm 0.954$ \\
4 & 0.938 & $0.832 \pm 0.978$ & 0.958 & $0.859 \pm 0.988$ \\
5 & 0.938 & $0.832 \pm 0.978$ & 0.917 & $0.804 \pm 0.967$ \\
6 & 0.958 & $0.859 \pm 0.988$ & 0.958 & $0.859 \pm 0.988$ \\
7 & 0.938 & $0.832 \pm 0.978$ & 0.917 & $0.804 \pm 0.967$ \\
8 & 0.979 & $0.890 \pm 0.996$ & 0.917 & $0.804 \pm 0.967$ \\
9 & 0.917 & $0.804 \pm 0.967$ & 0.917 & $0.804 \pm 0.967$ \\
10 & 0.896 & $0.778 \pm 0.954$ & 0.896 & $0.778 \pm 0.954$ \\
11 & 0.917 & $0.804 \pm 0.967$ & 0.979 & $0.890 \pm 0.996$ \\
\hline
\end{tabular}

Fuente: elaboración propia 


\section{Tabla 5 \\ Rúbrica analítica socioformativa para evaluar competencias investigativas en posgrado}

Nivel de
desempeño

Preformal

Receptivo

\section{西} y y Res

\begin{tabular}{l}
\hline Ítem \\
\hline \\
\\
estudiante es capaz de \\
identificar, describir y \\
explicar un problema de \\
investigación de su contexto \\
para proponer alternativas \\
de solución?
\end{tabular}

2. ¿En qué nivel el estudiante es capaz de recabar, seleccionar y organizar información válida y confiable para la fundamentación del problema identificado?

\section{A}

Estratégico Establece y argumenta un problema de investigación viable, original y relevante cuya Le resulta muy difícil identificar un problema de investigación, por lo que el
es quien le sugiere uno y necesita auxiliarle ampliamente en su formulación.

abordar, aunque requiere asistencia del asesor para delimitarlo y formularlo con total abordar,
claridad.

Resolutivo Consigue identificar y delimitar un problema de investigación tomando como referencia algún otro estudio que podría ser replicado en el propio contexto.

Autónomo Determina un problema de investigación importante y significativo para el propio contexto cuya resolución es viable y puede tener un impacto relevante en la comunidad. resolución puede tener incidencia tanto en su propio contexto como en el desarrollo del conocimiento científico sobre el tema.

Preformal Le es dificultoso recolectar y organizar información para fundamentar el problema de su investigación.

Receptivo Recolecta alguna información general sobre el problema de investigación, sin embargo, requiere asistencia del asesor para seleccionar fuentes confiables y pertinentes.

Resolutivo Logra recolectar y organizar suficiente información confiable para fundamentar el problema de investigación.

Autónomo Obtiene información válida y pertinente y la organiza debidamente para fundamentar el problema de investigación de manera completa y clara.

Estratégico Obtiene, información válida, confiable y actualizada, la registra y sistematiza, de manera que logra fundamentar el problema de investigación muy sólidamente.

Preformal Le resulta muy difícil o imposible identificar las variables que intervienen en el problema de investigación, y por tanto no logra formular preguntas y objetivos y/o hipótesis adecuados.

Receptivo Consigue identificar algunas variables relacionadas con el objeto de estudio, sin lograr distinguir su naturaleza y sus posibles relaciones, por lo que requiere la intervención del asesor para formular una pregunta y objetivos y/o hipótesis de investigación pertinentes.

3. ¿En qué nivel el estudiante es capaz de formular las preguntas, objetivos y/o hipótesis para seguir con el proceso de investigación?

4. ¿En qué nivel el estudiante es capaz de construir el marco teórico integrando diferentes
Resolutivo Identifica las variables que intervienen en el problema de investigación, las comprende, las clasifica y logra formular preguntas y objetivos y/o hipótesis sencillas, pero adecuados para trabajar sobre ellos.

Autónomo Identifica perfectamente las variables que intervienen en el problema de investigación y logra formular una pregunta pertinente y relevante, así como formular objetivos y/o hipótesis de investigación viables.

Estratégico Determina con precisión las variables de estudio, su naturaleza, clasificación y posible operacionalización, con las que construye varias preguntas de trabajo viables y elige la más pertinente para establecer objetivos y/o hipótesis de investigación muy relevantes.

Preformal Tiene dificultades para procesar información y se limita a construir el marco teórico exponiendo únicamente el concepto central de su objeto de estudio de forma muy elemental, mediante la transcripción de fragmentos de documentos relativos al tema.

Receptivo Consigue identificar los conceptos fundamentales del objeto de estudio y estructurar un texto suficientemente centrado en el tema de investigación. 
campos del saber en su investigación?

5. ¿En qué nivel el estudiante es capaz de exponer y argumentar la relevancia teórica, metodológica y práctica de la investigación?
Resolutivo Autónomo

Estratégico

Est

Preformal Tiene dificultad para identificar y expresar la importancia que reviste la investigación que pretende realizar.

Receptivo Logra expresar algunas ideas en torno a la importancia de la investigación que está realizando.

Resolutivo Explica de manera general la relevancia teórica, metodológica y práctica, de la investigación, a partir de algunas consideraciones sencillas y cercanas a la experiencia particular o a las aplicaciones básicas del tema estudiado.

Autónomo Explica y argumenta la relevancia teórica, metodológica y práctica, de la investigación a partir de datos de la propia experiencia y de la literatura científica específica del campo del conocimiento.

Estratégico Expone, de forma completa, clara y bien argumentada la relevancia teórica, metodológica y práctica, de la investigación, basándose en datos científicos multidisciplinarios, tanto históricos como contemporáneos y en las necesidades y problemas importantes del propio contexto.

Preformal Desconoce los paradigmas, tipos y diseños de investigación y sus características, por lo que no logra determinar estos aspectos de su proyecto de investigación.

Receptivo Logra definir el paradigma de su investigación en congruencia con los objetivos, así como ofrecer una explicación y justificación básica.

6. ¿En qué nivel el seleccionar el diseño de investigación?

Resolutivo

Determina el paradigma y el tipo de investigación, los explica basado en la literatura y los justifica con base en el objeto de estudio y los objetivos de la investigación.

Autónomo Elige, justifica y argumenta el paradigma, el tipo y el diseño de su investigación con precisión técnica y en perfecta congruencia con el objeto de estudio y las metas de investigación.

Estratégico Plantea distintas posibilidades metodológicas para abordar la investigación y elige el paradigma, el tipo y el diseño que resulta más pertinente y relevante, ofreciendo las explicaciones epistemológicas, teóricas y metodológicas que fundamentan su elección.

Preformal Le es dificultoso organizar y clasificar la información obtenida

Receptivo Realiza una muy básica organización y sistematización de los datos obtenidos en el trabajo de campo. Lo logra con cierta dificultad y requiriendo mucha asistencia por parte del asesor

7. ¿En qué nivel el estudiante es capaz de registrar y sistematizar los datos obtenidos en el trabajo de campo?

Resolutivo

Realiza la sistematización de los datos obtenidos en el trabajo de campo a partir de categorías de clasificación generales y empleando recursos sencillos como codificaciones gráficas, síntesis, tablas y fichas

Autónomo Sistematiza la información obtenida en el trabajo de campo a partir de categorías claramente establecidas en el marco teórico y agrega las que son necesarias. Emplea tanto recursos manuales (fichas, tabulaciones, etc.) como informáticos (bases de datos, hojas de cálculo, etc.)

Estratégico Realiza una sistematización de datos muy completa, precisa y clara, a partir de criterios y categorías relevantes para el objeto de estudio. Emplea recursos informáticos avanzados como software estadístico o de análisis cualitativo de textos.

8. ¿En qué nivel el estudiante es capaz de
Preformal Le es difícil identificar los datos clave y hacer las inferencias requeridas para responder la pregunta de investigación 
analizar los datos para estructurar la respuesta a la pregunta de investigación?

9. ¿En qué nivel el estudiante es capaz de interpretar los datos obtenidos para construir, exponer y fundamentar los resultados de la investigación?

10. ¿En qué nivel el estudiante es capaz de redactar el informe final (Tesis) del trabajo con precisión académicocientífica (redacción y ortografía) y técnica (normas APA vigente)?
Receptivo Requiere bastante ayuda por parte del asesor para identificar y jerarquizar los datos clave de forma adecuada para dar respuesta a la pregunta de investigación

Resolutivo Consigue realizar una buena reducción de los datos y conformar esquemas básicos para responder la pregunta de investigación.

Autónomo Realiza una esquematización clara y completa de los datos y logra inferir los ejes clave para responder la pregunta de investigación

Estratégico Construye un esquema integrador a partir de un análisis sistemático de los datos, construye los ejes clave para ofrecer una respuesta integral y novedosa a la pregunta de investigación

Preformal Le es difícil inferir los significados de la información obtenida. La exposición de los resultados se limita a una presentación de los datos.

Receptivo Consigue llegar a una incipiente interpretación de los datos y a estructurar una presentación suficientemente coherente de los resultados.

Resolutivo Logra hacer una buena interpretación de los datos y llega a unos resultados bien fundamentados.

Autónomo Logra interpretar los datos de forma muy completa. Llegando a resultados muy bien fundamentados teórica y empíricamente.

Estratégico Realiza una interpretación muy profunda e integral de los datos, con lo que llega a resultados relevantes y novedosos que formula y explica con sólidos fundamentos teóricos y empíricos.

Preformal Presenta un informe cuyo texto tiene problemas de organización, descuidos ortográficos e importantes inconsistencias técnicas.

Receptivo Logra estructurar un informe suficientemente completo y ordenado, pero con algunos descuidos literarios y técnicos.

Resolutivo Elabora un informe completo y correcto literaria y técnicamente.

Autónomo Elabora un informe completo en su estructura, con muy buena presentación y total precisión literaria y técnica.

Estratégico Produce un informe completo e integral en su estructura, excelente en su presentación, con absoluta precisión técnica y con perfección literaria en cuanto a ortografía, redacción y estilo.

Preformal Le es difícil establecer contacto con profesionales y especialistas del entorno cercano para solicitar apoyo en el desarrollo de su trabajo de investigación.

11. ¿En qué nivel el estudiante es capaz de obtener colaboración y apoyo de parte de personajes solventes profesional y académicamente para el desarrollo de su investigación?

\section{Receptivo}

Establece algunos contactos con profesionales y especialistas en el entorno cercano, para solicitar apoyo en el desarrollo de su trabajo de investigación.

Resolutivo Establece contacto con profesionales y académicos especialistas del entorno nacional y obtiene de ellos aportes relevantes para su trabajo de investigación

Autónomo Establece contacto con profesionales, académicos e investigadores especialistas a nivel nacional con quienes establece vínculos de apoyo académico y científico.

Estratégico Establece contacto con profesionales, académicos e investigadores especialistas a nivel nacional e internacional para incorporarse a redes de investigadores y académicos especializadas en los temas afines a su investigación.

Fuente: elaboración propia

\section{Grupo piloto}

Los resultados fueron favorables con respecto a la satisfacción con el instrumento, en específico se hace énfasis en que se precisa un buen grado de comprensión de las instrucciones, ítems y relevancia de las preguntas (Tabla 6). Respecto del análisis de consistencia interna evidenció una alta confiabilidad (Alfa de Cronbach: 0.832). 


\section{Tabla 6}

Análisis de satisfacción con el instrumento

\begin{tabular}{lcccc}
\hline \multicolumn{1}{c}{ Ítem } & $\begin{array}{c}\text { Bajo grado } \\
(\boldsymbol{\%})\end{array}$ & $\begin{array}{c}\text { Aceptable grado } \\
(\boldsymbol{\%})\end{array}$ & Buen grado (\%) & $\begin{array}{c}\text { Excelente grado } \\
(\boldsymbol{\%})\end{array}$ \\
\hline $\begin{array}{l}\text { Grado de comprensión de las } \\
\text { instrucciones }\end{array}$ & 5.882 & 11.765 & 47.059 & 35.294 \\
$\begin{array}{l}\text { Grado de comprensión de las } \\
\text { preguntas }\end{array}$ & 0.000 & 14.706 & 52.941 & 32.353 \\
$\begin{array}{l}\text { Grado de satisfacción del } \\
\text { instrumento }\end{array}$ & 0.000 & 11.765 & 47.059 & 41.176 \\
\begin{tabular}{l} 
Relevancia de las preguntas \\
\hline
\end{tabular} & 2.941 & 17.647 & 55.882 & 23.529 \\
\hline
\end{tabular}

Fuente: elaboración propia

\section{Discusión}

Las competencias investigativas son acciones generales que se asumen para reconocer, analizar y dar solución a los problemas reales que exige vinculación de los cuatro saberes; saber hacer, saber ser, saber conocer y sobre todo saber convivir; con idoneidad y ética (Aliaga-Pacora \& Luna-Menecio, 2020; Tobón, 2013), en los estudiantes de posgrado el desarrollo de las competencias investigativas son esenciales porque le va a permitir identificar diferentes problemas sociales (Martínez-Rodríguez \& Márquez-Delgado, 2014). A fin de modificar su contexto dentro de un trabajo colaborativo con idoneidad y considerando el proyecto de vida, Por consiguiente, conocer la calidad y el progreso de las competencias investigativas del estudiante de posgrado es sustancial. Empero estos instrumentos enfatizan la evaluación de la capacidad de manejo de las TIC, búsqueda de información y metodología. Por su parte la propuesta de Valdés-Cuervo et al. (2012) y Tapia et al. (2018) consideran las etapas del proceso de un trabajo de investigación.

La selección de los aspectos fue extensamente sostenida en los fundamentos del enfoque referido. Contemplando de vital importancia la evaluación de las competencias investigativas con una rúbrica analítica socioformativa que se conceptualiza como un acervo de estrategias que tiene como fin la formación integral de los ciudadanos, a través del trabajo colaborativo, dado que, trabajar con pares o expertos, fortalece la aptitud de no solo ser parte sino de constituir equipos científicos como efecto de una formación profesional científica (Ceballos-Almeraya \& Tobón, 
2019; Hernández-Mosqueda et al., 2016; Salazar-Gómez et al., 2018). Se enfatiza que permite valorar los aprendizajes y las producciones realizadas por los estudiantes o participantes, sin dejar de lado la clara identificación de la importancia de los contenidos y metas de los trabajos. Otro aspecto en común es el tipo de instrumento empleado, el cual en su mayoría son de tipo cuestionario con respuestas en escala tipo Likert.

Por lo expuesto, anteriormente, urge la necesidad de contar con un instrumento como la rúbrica socioformativa analítica, debido a que, ésta permite identificar los niveles específicos de logro, debido a que permite seccionar una actividad compleja en una más simple, distribuida en forma gradual, lo cual, facilita la autoevaluación de las habilidades y destrezas del estudiante. Además, su naturaleza descriptiva, la convierten en un instrumento que orienta el proceso evaluativo, originando una información de calidad sobre sus resultados (Montoya-López \& JuarezHernández, 2019).

En ese marco, la rúbrica socioformativa como instrumento de evaluación se conceptualiza como un grupo de estrategias que procura la formación de personas emprendedoras, y orienta a un sólido proyecto ético de vida y que a través del trabajo colaborativo busca dirimir problemas y alcanzar el desarrollo sustentable (Salas-Razo \& Juarez-Hernandez, 2017). En tal sentido, el instrumento propuesto, permitirá a los asesores de tesis identificar la calidad y el progreso de los estudiantes respecto al desarrollo de las competencias investigativas, evidenciados en el desarrollo del trabajo de tesis, debido a que presentan descriptores para los diferentes niveles de dominio (Marin-Garcia et al., 2015; Tobón et al., 2020). Estos niveles de dominio orientan al docente y a los estudiantes en su desarrollo de forma gradual, es decir, de elemental a lo complejo (Tobón, 2017). Además, los niveles de dominio permiten identificar en qué nivel se sitúa el estudiante, debido a que cada nivel posee descriptores que ayudan a determinar con precisión el nivel, conforme a la taxonomía socioformativa (preformal, receptivo, resolutivo, autónomo y estratégico (Tobón, 2017)

Con relación a los momentos metodológicos seguidos; en primer orden se advierte la validez de facie que estriba en una valoración de un conjunto de expertos que valoran; el término utilizado, claridad en las instrucciones, organización del instrumento, realizando subgerencias u observaciones en relación a la pertinencia, relevancia, comprensión y coherencia de los ítems (Juárez-Hernández \& Tobón, 2018). Es decir, se valora el contenido para que la persona evaluada comprenda adecuadamente los ítems del instrumento. Esta fase fue determinante para la 
optimización de la rúbrica analítica en la definición y configuración del constructo desde el marco de la socioformación y de la sociedad del conocimiento.

El segundo momento metodológico fue el análisis de validez de contenido, que según (Urrutia et al. (2014) así como Boluarte-Carbajal \& Kotaro-Tamari (2017) representa un primer nivel de validez que consiste en la calidad y precisión de un instrumento de investigación, y se relaciona con la obtención de evidencias válidas centradas en el contenido, la consistencia interna, las respuestas, el vínculo con otras variables. Además, facilita la identificación de las debilidades y fortalezas del instrumento, la toma de decisiones respecto a la adaptación, integración o eliminación de algún ítem.

Se determino la validez de contenido mediante la técnica juicio de expertos. Este método es un procedimiento que demanda interpretación y aplicación de sus resultados de forma adecuada, infalible y con toda la rigurosidad metodológica y estadística (Escobar-Pérez \& Cuervo-Martínez, 2008), de ahí la exigencia y rigurosidad en la caracterización, selección y numero de los expertos que van a integrar el juicio y el enfoque de este (cuantitativo-cualitativo) (Juárez-Hernández \& Tobón, 2018). Por ello, los jueces que participaron en el estudio son profesionales de amplio perfil, la mayoría con grado de maestro y doctor, y con experiencia en investigación. La valoración permitió reunir sugerencias para la optimización de la rúbrica, y realizar un análisis mediante el coeficiente de relevancia (V de Aiken), el cual permitió evidenciar la validez de contenido.

El tercer momento metodológico fue la aplicación de una encuesta de satisfacción sobre la rúbrica a un grupo piloto, denominado también piloteo del instrumento. Según HernándezSampieri et al. (2018) es importante aplicar el instrumento a una pequeña muestra de prueba para asegurar la pertinencia y eficacia del instrumento, así como también las condiciones de la aplicación y los procedimientos del instrumento. En esta investigación, el grupo piloto estuvo conformado por 38 asesores de tesis de la Escuela Universitaria de Posgrado, de la Universidad Nacional Federico Villarreal del Distrito de Lima Cercado. Los resultados fueron favorables con respecto a la satisfacción con el instrumento, en específico se hace énfasis en que se precisa un buen grado de comprensión de las instrucciones e ítems y relevancia de las preguntas.

Respecto del análisis de consistencia interna evidenció una alta confiabilidad (Alfa de Cronbach: 0.832). En este sentido, la confiabilidad de un instrumento se refiere a la exactitud de los resultados al aplicarse el instrumento en disímiles momentos (Carvajal et al., 2011). La investigación evidencia que el instrumento tiene valores convenientes de confiabilidad, que según 
Dominguez-Lara \& Rodriguez (2017) los valores de Alfa de Cronbach superiores a 0,7 significan un buen grado de consistencia interna de un instrumento.

Una limitación del presente estudio fue la falta del análisis de validez de constructo, la cual es considerada como el principal tipo de validez (Messick, 1980), la ausencia de este análisis se corresponde con el tamaño de muestra del grupo piloto ya que como lo refiere Costello \& Osborne (2005) se requiere de una muestra mínima superior a 70 participantes para llevar a cabo este análisis mediante el análisis factorial exploratorio. Lo anterior, motiva a la aplicación del instrumento a una muestra funcional para tal fin y proseguir con el esquema de validación.

\section{Conclusiones}

Se presenta un instrumento para evaluar las competencias investigativas de los estudiantes de posgrado, la cual integra 11 ítems comprendidos en cinco aspectos. El instrumento al estar conformado como rúbrica analítica socioformativa permitirá establecer el nivel de dominio de habilidades, destrezas y capacidades investigativas. Por su parte, las etapas metodológicas permitieron asegurar que el instrumento posee validez de contenido, es entendible para la población objetivo y confiable. Sin embargo, se requiere proseguir con el análisis de sus propiedades psicométricas (validez de constructo) para que el instrumento obtenga una calidad óptima.

\section{Referencias}

Aliaga-Pacora, A., \& Luna-Menecio, J. (2020). La construcción de competencias investigativas del docente de posgrado para lograr el desarrollo social sostenible. Revista Espacios, 41(20), 012. https://bit.ly/3e7qpaI

Balbo, J. (2000). Formación en competencas investigativas, un nuevo reto de las universidades. Universidad Nacional Experimental, 8, 1-16. https://bit.ly/2TzbGvZ

Boluarte-Carbajal, A., \& Kotaro-Tamari. (2017). Validez de contenido y confiabilidad interobservadores de Escala Integral Calidad de Vida. Revista de Psicologia (Peru), 35(2), 641666. https://doi.org/10.18800/psico.201702.009

Campos-Céspedes, J., Madriz Bermúdez, L., Brenes Matarrita, O. L., Rivera Sánchez, Y., \& Viales Sossa, M. (2012). Competencias investigativas en el personal académico de la Escuela de Ciencias de la Educación de la UNED, Costa Rica. UNED Research Journal, 4(2), 273-282. 
https://doi.org/10.22458/urj.v4i2.16

Cardona-Arias, J. A. (2019). Impacto de las prácticas profesionales sobre las competencias de investigación formativa en estudiantes de Microbiología de la Universidad de AntioquiaColombia. Revista Virtual Universidad Católica Del Norte, 56, 2-15. https://bit.ly/34G5hFF

Cardoso, E. O., \& Cerecedo, M. T. (2019). Assessment of the research competences of students in graduate courses in administration . Formacion Universitaria, 12(1), 35-44. https://doi.org/10.4067/S0718-50062019000100035

Carvajal, A., Centeno, C., Watson, R., Martínez, M., \& Sanz Rubiales, Á. (2011). ¿Cómo validar un instrumento de medida de la salud? In Anales del Sistema Sanitario de Navarr, 34(1), 6372. https://doi.org/10.4321/s1137-66272011000100007

Ceballos-Almeraya, J. M., \& Tobón, S. (2019). Validez de una rúbrica para medir competencias investigativas en pedagogía desde la socioformación Validity of a rubric to measure research competences in pedagogy from the socioformation. Atenas, 3(August), 1-17. https://bit.ly/2Jjrxgi

Costello, A. B., \& Osborne, J. W. (2005). Best practices in exploratory factor analysis: Four recommendations for getting the most from your analysis. Practical Assessment, Research and Evaluation, 10(7), 1-9. https://bit.ly/3jGV6Vp

Díaz-Barriga, Á. (2011). Competências em educação: Correntes de pensamento e implicações para o currículo e o trabalho no sala-de-aula. Revista Iberoamericana de Educación Superior, 2(5), 3-24. https://bit.ly/34H4ZOJ

Dominguez-Lara, S., \& Rodriguez, A. (2017). Índices estadísticos de modelos bifactor. Interacciones: Revista de Avances En Psicología, 3(2), 59-65. https://doi.org/10.24016/2017.v3n2.51

Escobar-Pérez, J., \& Cuervo-Martínez, Á. (2008). Validez De Contenido Y Juicio De Expertos: Una Aproximación a Su Utilización. Avances En Medición, 6 (January 2008), 27-36. https://bit.ly/35Jis88

Estrada, L. (2019). Evaluación del desarrollo de competencias investigativas: Un estudio en la formación inicial de docentes. Paradigma: Revista de Investigación Educativa, 26(41), 6992. https://doi.org/10.5377/paradigma.v26i41.7976

Fraile, J., Pardo, R., \& Panadero, E. (2017). ¿Cómo emplear las rúbricas para implementar una verdadera evaluación formativa? Revista Complutense de Educacion, 28(4), 1321-1334. 
https://doi.org/10.5209/RCED.51915

George-Reyes, C., \& Ramírez-Martinell, A. (2019). Competencias investigativas y saberes digitales de estudiantes de Posgrado en la modalidad virtual. Certiuni Journal, 0(5), 65-78. https://bit.ly/34FO5Qv

Gheller, S. (2002). Estrategia de intervención para resolver el síndrome Todo menos tesis; en el componente docente de la Escuela de educación de la Universidad Central de Venezuela. Anales de La Universidad Metropolitana, 2(2), 13-32. https://dialnet.unirioja.es/servlet/articulo?codigo $=4004137$

Grijalva-Verdugo, A. A., \& Zazueta, M. L. U. (2017). Cultura científica desde la universidad. Evaluación de la competencia investigativa en estudiantes de Verano Científico. Education in the Knowledge Society (EKS), 18(3), 15. https://doi.org/10.14201/eks20171831535

Guajardo, C., Cadena, G., María, D., Romero, R., \& Virginia, E. (2011). Las competencias en investigación como puentes cognitivos para un aprendizaje significativo, 16(77), 55. https://www.redalyc.org/pdf/1995/199520010084.pdf

Gutiérrez-Castillo, J.-J., Cabero-Almenara, J., \& Estrada-Vidal, L. I. (2017). Design and validation of an instrument for evaluation of digital competence of University student . Espacios, 38(10), 16. https://bit.ly/34Gj3bq

Haynes, S. N., Richard, D., \& Kubany, E. S. (1995). Content validity in psychological assessment: A functional approach to concepts and methods. Psychological Assessment, 7(3), 238. https://bit.ly/32cBO19

Hernández-Mosqueda, J. S., Tobón-Tobón, S., \& Guerrero-Rosas, G. (2016). Hacia una evaluación integral del desempeño: las rúbricas socioformativas. $R a$ Ximhai, 12(6), 359-376. https://www.redalyc.org/pdf/461/46148194025.pdf

Hernández-Sampieri, R., Méndez, S., \& Mendoza, C. P. (2018). Centro de recursos en línea de la obra Metodología de la investigación: Las rutas cuantitativa, cualitativa y mixta. Ciudad de México. https://bit.ly/35K18Cd

Juárez-Hernández, L. G., \& Tobón, S. (2018). Análisis de los elementos implícitos en la validación de contenido de un instrumento de investigación. Revista Espacios, 39(53), 1-23. http://www.revistaespacios.com/cited2017/cited2017-23.pdf

Koller, I., Levenson, M. R., \& Glück, J. (2017). What do you think you are measuring? A mixedmethods procedure for assessing the content validity of test items and theory-based scaling. 
$\begin{array}{llll}\text { Frontiers } & \text { in } & \text { Psychology, } & 8,\end{array}$ https://www.frontiersin.org/articles/10.3389/fpsyg.2017.00126/full

Luque, E. H., Estrada, V., \& Keeling, M. (2018). Perspectivas y desafíos de la gestión del conocimiento y la competencia investigativa en la educación del posgrado. 6(1). http://uceciencia.edu.do/index.php/OJS/article/view/123

Marin-Garcia, J. A., Ramirez Bayarri, L., \& Atares Huerta, L. (2015). Protocol: Comparing advantages and disadvantages of Rating Scales, Behavior Observation Scales and Paired Comparison Scales for behavior assessment of competencies in workers. A systematic literature review. WPOM-Working Papers on Operations Management, 6(2), 49. https://doi.org/10.4995/wpom.v6i2.4032

Martínez-Rodríguez, D., \& Márquez-Delgado, D. (2014). Las habilidades investigativas como eje transversal de la formación para la investigación. Tendencias Pedagógicas, 24(24), 347-360. https://repositorio.uam.es/handle/10486/663144

Martínez-Valdés, M. G., \& Juárez-Hernández, L. G. (2019). Diseño y validación de un instrumento para evaluar la formación en sostenibilidad en estudiantes de educación superior. IE Revista de Investigación Educativa de La REDIECH, 10(19), 37. https://doi.org/10.33010/ie_rie_rediech.v10i19.501

Mendoza Mendoza, J. G., \& Garza, J. B. (2009). La medición en el proceso de investigación científica: Evaluación de validez de contenido y confiabilidad (Measurement in the scientific research process: Content validity and reliability evaluation). Innovaciones de Negocios, 6(11), 17-32. http://eprints.uanl.mx/12508/

Messick, S. (1980). Test validity and the ethics of assessment. American Psychologist, 35(11), 1012. https://psycnet.apa.org/record/1981-27017-001

Mgquba, S. K., \& Underwood, P. G. (2016). Enhancing information research and learning skills through e-learning: the case of Monash University Library. South African Journal of Libraries and Information Science, 81(2), 39-45. https://doi.org/10.7553/81-2-1561

Montoya-López, L., \& Juárez-Hernández, L. G. (2019). Validación de contenido de un instrumento para la calidad educativa mediante la metodología instruccional ADOIVA. Atenas, 3(47), 5269. Recuperado de: https://atenas.reduniv.edu.cu/index.php/atenas/article/view/489

Moros-Briceño, J. A. (2018). Evaluación de las competencias investigativas en los estudiantes de maestría de la Universidad Nacional Experimental del Táchira mediante el uso de modelos de 
regresión multinivel. ECOMATEMATICO, $\quad$ 51-64. https://doi.org/10.22463/17948231.1670

Parrado-Lozano, Y. M., Sáenz Montoya, X., Soto Lesmes, V. I., Guáqueta Parada, S. R., Amaya Rey, P., Caro Castillo, C. V., Parra Vargas, M., \& Triana Restrepo, M. C. (2015). Validez de dos instrumentos para medir la relación interpersonal de la enfermera con el paciente y su familia en la unidad de cuidado intensivo. Investigación En Enfermería: Imagen y Desarrollo, 18(1), 115. https://doi.org/10.11144/javeriana.ie18-1.vimr

Penfield, R. D., \& Giacobbi, Jr, P. R. (2004). Applying a score confi dence interval to Aiken's item content-relevance index. Measurement in Physical Education and Exercise Science, 8, 213-225

Reiban, R., De la Rosa, H., \& Zeballos, J. (2017). Competencias investigativas en la Educación Superior. Competencias Investigativas En La Educación Superior Revista Publicando, 4(10), 395-405. https://n9.cl/dabrw

Rizo-García, M. (2012). Enseñar a investigar investigando. Experiencias de investigación en comunicación con estudiantes de la Licenciatura en Comunicación y Cultura de. 20-39. https://bit.ly/3jCKDdP

Salas-Razo, G., \& Juarez-Hernandez, L. G. (2017). Hacia un modelo de desarrollo rural integral sustentable basado en la sociedad del conocimiento. Espacios, 39(53), 9. http://www.redalyc.org/articulo.oa?id=181351615008

Salazar Gómez, E., Tobón, S., \& Juárez Hernández, L. G. (2018). Diseño y validación de una rúbrica de evaluación de las competencias digitales desde la socioformación. Apuntes Universitarios, 8(3), 24-42. https://doi.org/10.17162/au.v8i3.329

Tapia, C., Cardona, S. A., \& Vázquez, H. (2018). Research competences in postgraduate: Experience of an online course | Las competencias investigativas en posgrado: Experiencia de un curso en línea. 20. http://www.revistaespacios.com/cited2017/cited2017-20.pdf

Tobón, S. (2017b). Evaluación socioformativa. Estrategias e instrumentos. Mount Dora (USA): Kresearch. ISBN 978-1-945721-26 -7. https://cutt.ly/vu3RP8

Tobon, S., Juarez-Hernandez, L. G., Herrera-Meza, S. R., \& Nunez, C. (2020). Assessing school principal leadership practices. Validity and reliability of a rubric. Educacion XX1, 23(2), 187210. https://doi.org/10.5944/educxx1.23894 
Tobón, S. (2008). La formación basada en competencias en la educación superior: el enfoque complejo. México: Universidad Autónoma de Guadalajara. https://bit.ly/3jFxCA0

Tobón, S. (2013). Ejes esenciales de la sociedad del conocimiento y la socioformación. Mount Dora, USA: Kresearch. Disponible en: https://cife.edu.mx/recursos/2018/09/06/ejesesenciales-de-la-sociedad-del-conocimiento-y-la-socioformacion/

Urrutia, M., Barrios, S., Gutiérrez, M., \& Mayorga, M. (2014). Métodos óptimos para determinar validez de contenido. Revista Cubana de Educacion Medica Superior, 28(3), 547-558. https://bit.ly/31SQZ2I

Valdés-Cuervo, A., Carlos Martínez, E., Vera Noriega, J., \& Montoya Verduro, G. (2012). Propiedades psicométricas de un instrumento para medir las relaciones familiares en adolescentes intelectualmente sobresalientes. Pensamiento Psicológico, 1, 39-50. https://doi.org/10.11144/177

Vázquez-Antonio, J., Tobón, S., Vázquez-Antonio, J. M., Guzmán, C. E., \& Herrera-Meza, S. R. (2018). Diseño y validez de contenido de una rúbrica socioformativa para evaluar la tesis de investigación en la Educación Normal. Revista Espacios, 39(53). http://www.revistaespacios.com/cited2017/cited2017-28.html

Verhoeven, J. C., Heerwegh, D., \& De Wit, K. (2016). ICT learning experience and research orientation as predictors of ICT skills and the ICT use of university students. Education and Information Technologies, 21(1). https://doi.org/10.1007/s10639-014-9310-3 